CRÍTICA, Revista Hispanoamericana de Filosofía

Vol. XXXI, No. 93 (diciembre 1999): 21-47

\title{
TABLAS SEMÁNTICAS Y METALÓGICA (EL CASO DE LA LÓGICA DE SEGUNDO ORDEN)*
}

\author{
Ángel Nepomuceno FernándeZ \\ Departamento de Filosofía y Lógica y Filosofía de la Ciencia \\ Universidad de Sevilla
}

\section{Introducción}

El método de Beth, conocido como el método de las tablas semánticas, ${ }^{1}$ permite la demostración del teorema de satisfacción cuya formulación puede ser la siguiente:

Si $\Gamma$ es un conjunto consistente de sentencias de primer orden, entonces $\Gamma$ es satisfacible en un universo finito o infinito numerable.

A partir de este resultado, Henkin ${ }^{2}$ demuestra la completitud de la lógica de predicados de primer orden y señala que el método tiene aplicación en el estudio de lógica de orden superior. Es decir, este resultado puede ser extendido a lógicas superiores, en particular a la de segundo orden, de modo que de éstas, aunque con ciertas restricciones, se puede probar alguna forma de completitud.

* Subvencionado parcialmente por el Proyecto de Investigación PB96-1301-C05-03 del Ministerio Español de Educación y Cultura.

1 También llamado de los árboles semánticos, por la presentación arbórea invertida que se puede hacer al construirlas. Usaremos ambos apelativos indistintamente.

${ }^{2}$ Véase Henkin (1949) y Henkin (1950). 
Esquemáticamente, el método de la prueba de este teorema, para primer orden, consiste en la extensión de $\Gamma$ a un conjunto $\Gamma_{\omega}$ tal que sea máximamente consistente - incluir una nueva fórmula lo haría inconsistente - y ejemplificado —si una fórmula del tipo $\exists x \varphi \in \Gamma$, entonces $\varphi(b / x)^{3} \in \Gamma$; contiene alguna instancia de la matriz para un parámetro (constante individual) del lenguaje. Se define además una estructura interpretativa para dicho conjunto extendido, cuyo universo de discurso es el de ciertas clases de equivalencia de parámetros, por lo que es finito o, a lo sumo, numerable; se prueba que dicha estructura satisface todas las sentencias de $\Gamma_{\omega} \mathrm{y}$, en consecuencia, también satisface las del conjunto inicial $\Gamma$.

La completitud resulta entonces ser un corolario del teorema de satisfacción: si $\Gamma \models \varphi$, entonces $\Gamma \cup\{\neg \varphi\}$ es no satisfacible, y por contraposición, $\Gamma \cup\{\neg \varphi\}$ es no consistente y, en consecuencia, $\Gamma \vdash \varphi$.

Aunque el método de árboles semánticos tiene algunos antecedentes, como, por ejemplo en Herbrand, fue elaborado por Beth, quien en $1955^{4}$ ideó una prueba del mismo teorema de satisfacción y, por lo tanto, de la completitud de la lógica de predicados de primer orden, que tiene sobre el método de Henkin la ventaja de ser más económica.

Para la nueva demostración se definen los árboles de Beth y se prueba que si $\Gamma$ es satisfacible, entonces el árbol de Beth de $\Gamma$ es abierto; en segundo lugar, se prueba que si el árbol de Beth de $\Gamma$ es cerrado, entonces $\Gamma$ es no consistente. De esto último se sigue, por contraposición, que si $\Gamma$ es consistente, entonces el árbol semántico de Beth de $\Gamma$ no es cerrado (es abierto). En tercer lugar, se

3 Sustitución de $x$ por $b$ en $\varphi$, según se define más adelante. no. 18.

4 Beth (1955); hay una versión en español en Cuadernos Teorema, 
prueba que si el árbol de Beth de $\Gamma$ no es cerrado, entonces $\Gamma$ es satisfacible en un universo finito o infinito numerable.

El último resultado junto con el anterior constituyen una versión del teorema de satisfacción, y su prueba es muy similar a la de Henkin: a partir de las sentencias de cualquiera de las ramas del árbol que haya quedado abierta se define una estructura interpretativa y se prueba que la misma satisface todas las sentencias de las ramas en cuestión.

Se advierte, pues, una indudable utilidad del método en lógica de predicados de primer orden. ¿Es asimismo aplicable en lógica de predicados de orden superior, en particular, en la de segundo orden? Si para comprender mejor la naturaleza de la cuantificación hemos de afrontar los problemas que ésta suscita cuando se extiende a variables de predicado, tratándose de un asunto nada trivial, la respuesta a dicha cuestión no puede ser una espontánea afirmación de que tal método sería aplicable, mutatis mutandi, a orden superior; como tampoco una apresurada negativa dada la incompletitud esencial de la lógica de segundo orden.

En este trabajo presentamos el procedimiento de tablas semánticas de Beth (y alguna modificación del mismo) en primer orden. A continuación se describe brevemente un lenguaje formal de segundo orden, destacando ciertas clases de fórmulas. En el apartado subsiguiente se trabaja con tablas aplicadas a (matrices de) sentencias de estas clases, definiendo reglas adicionales para tratar una de las clases en cuestión. Por último, se presenta una serie de observaciones sobre estas aplicaciones. A lo largo del texto aparecen los teoremas (y un lema) que hemos considerado relevantes; sus demostraciones se incluyen esquemáticamente, o se indica dónde pueden hallarse, salvo en el caso del teorema 9 y el lema necesario para probarlo, las cuales (y las definiciones necesarias), por su extensión, se han desarrollado en un apéndice. 


\section{Reglas de formación de árboles de primer orden}

Se han de especificar las nociones necesarias para las tablas semánticas de primer orden. A estos efectos, hacemos uso de $L^{1}$, un lenguaje formal de primer orden sin identidad ni functores y con constantes individuales y predicativas (denominados parámetros y relatores, respectivamente). Un árbol de Beth de sentencias es un conjunto de sucesiones de sentencias de $L^{1}$; estas sucesiones se denominan ramas, generadas a partir de un conjunto no vacío de sentencias de $L^{1}$ por aplicación a éstas de las reglas detalladas más adelante así como a las sentencias no elementales resultantes. Dispuestas sucesivamente las sentencias generadoras (que son las asunciones iniciales), diremos que no están convenientemente marcadas si no son elementales, constituyendo el comienzo de una rama; a cada sentencia no elemental no convenientemente marcada se aplicará la regla correspondiente, escribiendo a continuación las sentencias resultantes y se marcará con determinado signo lógico como subíndice (excepto cuando se trate de un cuantificador, en cuyo caso se anotarán los parámetros utilizados); el proceso se continúa hasta que todas las sentencias no elementales queden marcadas o aparezca un par de contradicción, entendiendo por tal las sentencias elementales $\delta$ y $\delta^{\prime}$ de manera que una es negación de la otra.

Sea $\varphi$ una sentencia no elemental no convenientemente marcada; las reglas se definen en función del grado de complejidad de $\varphi$ :

1. si $\varphi$ es $\neg \psi$, entonces
(a) si $\psi$ es $\neg \beta$, entonces se añade $\beta$;
(b) si $\psi$ es $\beta \wedge \gamma$, entonces se añade $\neg \beta \vee \neg \gamma$;
(c) si $\psi$ es $\beta \vee \gamma$, entonces se añade $\neg \beta \wedge \neg \gamma$;
(d) si $\psi$ es $\beta \rightarrow \gamma$, entonces se añade $\beta \wedge \neg \gamma$; 
(e) si $\psi$ es $\exists x \beta$, entonces se añade $\forall x \neg \beta$;

(f) si $\psi$ es $\forall x \beta$, entonces se añade $\exists x \neg \beta$;

además se marca $\varphi$ con $\neg$ como subíndice.

2. Si $\varphi$ es $\psi \wedge \beta$, entonces se añaden consecutivamente $\psi$ y $\beta$, y se marca $\varphi$ con $\wedge$.

3. Si $\varphi$ es $\psi \vee \beta$, entonces se abren dos subramas, una con $\psi$ y otra con $\beta$, se marca $\varphi$ con $\vee$, y decimos que $\varphi$ es un punto de bifurcación.

4. Si $\varphi$ es $\psi \rightarrow \beta$, entonces se abren dos subramas, una con $\neg \varphi$ y otra con $\beta$, se marca $\varphi$ con $\rightarrow$, y, asimismo, decimos que $\varphi$ es un punto de bifurcación.

5. Si $\varphi$ es $\exists x \psi$, entonces se añade $\psi\left(b_{k+1} / x\right)$, siendo $b_{k+1}$ el parámetro de menor subíndice que no ocurría antes en la rama (es decir, únicamente ocurrían $b_{i}$, para $i \leq k)$, y se marca $\varphi$ con dicho parámetro.

6. Si $\varphi$ es $\forall x \psi$, entonces se añaden $\psi\left(b_{1} / x\right), \psi\left(b_{2} / x\right), \ldots$, $\psi\left(b_{n} / x\right)$, siendo $b_{1}, \ldots, b_{n}$ todos los parámetros que ocurren en fórmulas de la rama.

Cuando en una rama aparece un par de contradicción - en cuyo caso se detiene el proceso, como se ha indicado- se dice que tal rama es cerrada. Una rama está acabada si es cerrada o, en otro caso, si todas sus fórmulas no elementales están convenientemente marcadas. Un árbol es cerrado cuando todas sus ramas son cerradas. Un árbol está acabado cuando todas sus ramas están acabadas. La propiedad fundamental de los árboles semánticos se expresa en el siguiente teorema cuya demostración omitimos para abreviar. ${ }^{5}$

${ }^{5}$ En Beth (1955) aparece la prueba del "teorema de árboles"; una demostración del teorema tal como se formula a continuación, aparece 
Teorema 1: Dados un conjunto $\Gamma$ de sentencias y una sentencia $\varphi \in L^{1}, \Gamma \models \varphi$ si y sólo si el árbol de $\Gamma \cup\{\neg \varphi\}$ es cerrado.

También se puede enunciar diciendo que $\varphi$ es satisfacible si y sólo si el árbol de $\{\varphi\}$ es abierto. A partir de una rama abierta se define una $L^{1}$-estructura que satisface todas las sentencias de la rama en cuestión. Esta $L^{1}$-estructura representa una clase de $L^{1}$-estructuras que satisfacen $\varphi$.

Además de los árboles de BETH, tomamos los árboles $\exists$ modificados ${ }^{6}$ obtenidos mediante una ligera modificación de la definición del árbol respecto de las sentencias a que dan lugar las fórmulas de la forma $\exists x \varphi$. Veamos la definición de la cláusula correspondiente en un caso y otro:

1. вETH. Se trata de la regla 5 citada: Si en una rama aparece una fórmula de la forma $\exists x \psi$ y está convenientemente marcada, entonces aparece también $\psi\left(b_{k+1} / x\right)$, siendo $b_{k}, k \geq 1$, el último parámetro que ocurría en la rama (antes de aplicar la regla 5).

2. $\exists$-MODIFICADOS. La anterior regla 5 se reformula de la siguiente manera: $\operatorname{Si} \varphi$ es $\exists x \psi$, entonces se abren subramas cada una de ellas con $\psi\left(b_{i} / x\right)$, para cada $i \leq k+1$, siendo $b_{k}, k \geq 0$, el último parámetro

en Nepomuceno (1995), pp. 45-49 y 58-60 — para lógica proposicional y para lógica de predicados de primer orden, respectivamente.

6 Díaz (1993) y Boolos (1984) idearon un tratamiento especial para fórmulas que generan árboles infinitos pero que son satisfacibles en dominios finitos. En Díaz (1993, p. 43) aparece una breve nota acerca del momento en que aparece tal tratamiento.

En este punto, como en otros a lo largo del trabajo, hemos de reconocer la deuda contraída con las ideas expuestas en Díaz (1987) y Díaz (1993) (así como las incluidas en documentos inéditos del mismo autor), especialmente las nociones de $\Delta$-fórmulas y las reglas especiales para determinados lenguajes. 
que ocurría en la rama (antes de la aplicación de esta regla) y se marca $\varphi$ con $\left|b_{1}\right|, \ldots,\left|b_{k}\right|, b_{k+1}{ }^{7}$

Los árboles obtenidos aplicando esta nueva regla poseen ciertas ventajas sobre los de Beth. Veámoslo en un sencillo ejemplo: Sea la sentencia $\forall x \exists y R x y$; el árbol de Beth tendría una única rama infinita, pero esta fórmula es satisfacible en un universo de un único individuo. En el árbol $\exists$-modificado, habría una rama con exactamente las siguientes sentencias:

$$
<\forall x \exists y R x y, \exists y R \mathbf{a}_{1} y, R \mathbf{a}_{1} \mathbf{a}_{1}>,
$$

la cual permite definir una $L^{1}$-estructura que satisface todas las fórmulas de dicha rama, con tal de que se interpreten el primer parámetro y el relator de la manera adecuada, aunque también contenga ramas infinitas, como

$$
<\forall x \exists y R x y, \exists y R \mathbf{a}_{1} y, R \mathbf{a}_{1} \mathbf{a}_{2}, \exists y R \mathbf{a}_{2} y, R \mathbf{a}_{2} \mathbf{a}_{3}, \ldots>
$$

\section{Un lenguaje formal de segundo orden}

$L^{2}$ es un lenguaje formal de segundo orden obtenido a partir de $L^{1}$, incluyendo variables predicativas y no restringiendo la cuantificación a las variables individuales. Así pues, si $\varphi$ es una fórmula de $L^{2}$ y $s$ es una variable de cualquier tipo (individual o predicativa de cierta aridad), entonces $\exists s \varphi, \forall s \varphi$ son fórmulas, y se dice que $s$ es el sufijo del cuantificador correspondiente. Las fórmulas de $L^{2}$ que

7 De este modo, las marcas $\left|b_{i}\right|, i \leq k$ expresan que se trata de ramas obtenidas por la nueva regla, mientras que la marca $b_{k+1}$ indica que se trata de una rama correspondiente a un árbol de Beth. 
carezcan de variables libres serán denominadas sentencias, como es usual.

Sea $\varphi \in L^{2}$, definimos $\varphi(r / s)$, donde $s$ es una variable y $r$ una variable individual o parámetro, si $s$ es individual, o bien una variable predicativa o relator de la misma aridad que $s$, si ésta es predicativa, según las cláusulas:

1. Si $s$ no ocurre, o no ocurre libre, en $\varphi$, entonces $\varphi(r / s)$ es $\varphi$.

2. Si $r$ es sufijo de un cuantificador bajo cuyo alcance cae $s$, entonces $\varphi(r / s)$ es $\varphi$.

3 . En otro caso, $\varphi(r / s)$ es la fórmula resultante de sustituir cada ocurrencia libre de $s$ en $\varphi$ por $r$.

Para representar la sustitución en $\varphi$ de $s_{1}$ y $s_{2}$ por $r_{1}$ y $r_{2}$ - siempre que sean del tipo correspondiente- se anotará $\varphi\left(r_{1}, r_{2} / s_{1}, s_{2}\right)$; en general, para sustitución de $n \geq 1$ términos, o sustitución simultánea, la notación es

$$
\varphi\left(r_{1}, \ldots, r_{n} / s_{1}, \ldots, s_{n}\right) .
$$

La semántica de $L^{2}$ queda establecida de la siguiente manera: Una $L^{2}$-estructura viene dada por un dominio o universo de discurso y una función interpretación:

$$
M=<D, \Im>
$$

en donde $\Im$ representa la denominada función interpretación, definida con dominio en los parámetros y relatores y rango en $D$ (y en los predicados y relaciones definidos en D) de manera que

1. Para cada parámetro $b, \Im(b) \in D$. 
2. Para cada relator $n$-ádico $R, \Im(R) \in \mathcal{P}\left(D^{n}\right)$, para todo $n \geq 1$.

La noción de satisfacción y las restantes nociones semánticas (validez en un dominio, validez universal, etc.) son las usuales. Si $\varphi \in L^{2}$ es una sentencia $M \models \varphi$ indica que $M$ satisface dicha sentencia; si $\Gamma$ es un conjunto de sentencias, $\Gamma \models \varphi$ indica que $\varphi$ es consecuencia lógica de $\Gamma$.

Podemos considerar que los relatores de $L^{1}$ son también relatores de $L^{2}$. Asimismo, cada fórmula de $L^{1}$ como una fórmula de $L^{2}$. En general, si $\varphi \in L^{2}, X_{1}, \ldots, X_{k}$, son sus variables predicativas, $R_{1}, \ldots, R_{k}$ son relatores tales que la aridad de $R_{i}$ es la misma que la de $X_{i}$, para todo $i \leq k$, y se verifica que

$$
\varphi\left(R_{1}, \ldots, R_{k} / X_{1}, \ldots, X_{k}\right) \in L^{1},
$$

entonces diremos que $\varphi$ es esencialmente de primer orden.

Respecto de fórmulas de $L^{2}$ en forma prenexa, el teorema de Zykov establece:

Para toda sentencia $\varphi \in L^{2}$ se puede obtener una sentencia $\psi$ en forma prenexa en la que los cuantificadores predicativos preceden a los cuantificadores individuales, tales que $\models \varphi \leftrightarrow \psi^{8}$

La demostración requiere la adopción de la semántica con axioma de elección. Por ahora, probadas una serie de equivalencias previas, lo que omitimos en aras de la brevedad, se obtiene:

${ }^{8} \varphi \leftrightarrow \psi$ se puede considerar abreviatura de $(\varphi \rightarrow \psi) \wedge(\psi \rightarrow \varphi)$ (de otro modo, fácilmente se redefiniría $L$ conteniendo este signo). La demostración original de Zykov (1956), es relativa a orden superior; en Hilbert-Ackermann (1962) aparece un resumen de su adaptación a segundo orden. 
Teorema 2: Para toda $\varphi \in L^{2}$ se puede hallar una fórmula $\psi$ en forma prenexa tal que $\models \varphi \leftrightarrow \psi$.

La demostración es análoga a la correspondiente a primer orden. ${ }^{9}$.

Definimos las siguientes clases de fórmulas:

1. $\sum_{0}^{1}=\Pi_{0}^{1}$, todas las fórmulas de $L^{2}$ que son esencialmente de primer orden;

2. para cada $n \geq 1, \sum_{n+1}^{1}=\left\{\exists X_{1}, \ldots, X_{m} \varphi: \varphi \in\right.$ $\left.\Pi_{n}^{1}, m \geq 1\right\}$,

3. $\Pi_{n+1}^{1}=\left\{\forall X_{1}, \ldots, X_{m} \varphi: \varphi \in \sum_{n}^{1}, m \geq 1\right\}$.

Son de cierto interés las clases $\sum_{1}^{1}$ y $\Pi_{1}^{1}$. En particular, dadas las formas prenexas de sentencias de $L^{2}$, nos interesan las clases siguientes:

1. $\sum_{1}^{1}$. Fórmulas con prefijo integrado por cuantificadores existenciales con sufijos predicativos y matriz esencialmente de primer orden;

2. $\Pi_{1}^{1}$. Fórmulas con prefijo integrado por cuantificadores universales con sufijos predicativos y matriz esencialmente de primer orden;

3. inversas de Zykov (IZ). $\varphi \in I Z$ si y sólo si $\varphi$ es de la forma $\mathcal{Q}_{1} \mathcal{Q}_{2} \psi$, en donde $\mathcal{Q}_{1}$ es un prefijo cuantificacional con sufijos individuales y $\mathcal{Q}_{2}$ es un prefijo cuantificacional con sufijos predicativos, no ocurriendo en $\psi$ ningún cuantificador.

Naturalmente no son éstas las únicas clases posibles de sentencias de $L^{2}$, pero el estudio de otras no constituye el objeto del presente trabajo.

9 Véase nota precedente 
4. Árboles de primer orden y las clases $\sum_{1}^{1}, \Pi_{1}^{1}$ e IZ

Antes de continuar, necesitamos de algunos resultados de la lógica de primer orden. Decimos que una sentencia $\varphi \in L^{1}$ es $n$-satisfacible, para $n \geq 1$, si y sólo si existe $M=$ $<D, \Im>$ tal que $|D|=n$ y $M \models \varphi$. Por otra parte, decimos que $\varphi$ es $n$-válida si y sólo si para toda $L^{1}$-estructura $M$ definida con $D$ como universo de discurso, $|D|=n$ y $M \models \varphi$.

A partir de estas nociones, se puede probar el siguiente teorema: ${ }^{10}$

Teorema 3: Si una sentencia $\varphi \in L^{1}$ es n-satisfacible, para $n \geq 1$, entonces es $m$-satisfacible, para todo $m \geq n$. Si es n-válida, entonces es $k$-válida, para todo $k \leq n$.

En efecto, sea $M=\langle D, \Im\rangle$ tal que $|D|=n$ y $M \models \varphi$. Sea $D^{*}$ tal que $|D| \leq\left|D^{*}\right|=m$ y $D^{\prime} \subseteq D^{*}$ tal que $|D|=$ $\left|D^{\prime}\right|=n$. Sea $f_{1}$ una función biunívoca de $D^{\prime}$ en $D$, $\mathbf{a}_{1}$ un elemento de $D^{\prime}$ escogido de una vez por todas. Sea $f$ una función de $D^{*}$ a $D$ tal que para cada $\mathbf{a} \in D^{*}$, si $\mathbf{a} \in D^{\prime}$, entonces $f(\mathbf{a})=f_{1}(\mathbf{a})$ y en caso contrario $f(\mathbf{a})=f\left(\mathbf{a}_{1}\right)$. La función $f$ nos permite definir una nueva función $h$, con dominio en el conjunto de los relatores $n$-ádicos de $L^{1}$ y rango en $\mathcal{P}\left(D^{* n}\right)$, según la regla:

$$
h(R)=\left\{<\mathbf{b}_{1}, \ldots, \mathbf{b}_{n}>\in D^{* n}:<f\left(\mathbf{b}_{1}\right), \ldots, f\left(\mathbf{b}_{n}\right)>\in \Im(R)\right\} .
$$

Entonces, por inducción sobre el grado de complejidad de $\varphi$, se prueba que es definible $\Im^{*}$, tal que para cada relator $R$ de $L^{1}, \Im^{*}(R)=h(R)$; se obtiene así $M^{*}=$ $<D^{*}, \Im^{*}>$, tal que

$$
M \models \varphi \text { si y sólo si } M^{*} \models \varphi .
$$

10 Relacionado con los de Löwenheim-Skolem. 
En cuanto a la segunda parte del teorema, sea $\varphi$ una sentencia $n$-válida; supongamos que no se verifica que sea $k$-válida para cierto $k \leq n$; entonces, por definición, $\neg \varphi$ sería $k$-satisfacible, por lo que sería también $n$-satisfacible, en contra de la hipótesis inicial. $\square$.

Teorema 4: Un conjunto de sentencias $\Gamma \subseteq L^{1}$ es $n$ satisfacible, para un cierto $n \geq 1$, si y sólo si el árbol $\exists$ modificado de $\Gamma$ posee una rama abierta en la que ocurren sólo $n$ parámetros.

La prueba se realiza partiendo de la hipótesis de que existe $M=\langle D, \Im\rangle$ tal que $|D|=n \geq 1$ y $M \models \varphi$, para cada $\varphi \in \Gamma$. Sea $\Gamma^{\prime}$ el conjunto de sentencias obtenido desde $\Gamma$ al sustituir las distintas constantes individuales de las sentencias de $\Gamma$ que tengan el mismo valor para la función $\Im$ por una de ellas —así, en $\Gamma^{\prime}$ no hay ningún par de parámetros $a$ y $b$ tales que $\Im(a)=\Im(b)$. Obviamente, $M$ satisface cada fórmula de $\Gamma^{\prime}$. Entonces, por inducción sobre el nivel de las sentencias del árbol (número de aplicación de las reglas), se demuestra que hay al menos una rama $\mathcal{R}$ no cerrada en la cual ocurren justamente $n$ parámetros y que $M^{\prime}$ satisface las sentencias de la rama, siendo $M^{\prime}$ tal que difiere de $M$ a lo sumo en cuanto a los valores para la función interpretación de los parámetros que ocurran en sentencias de $\mathcal{R}$ y que no ocurren en $\Gamma^{\prime}$.

En efecto, en el primer nivel hay una fórmula que no constituye un par de contradicción con ninguna otra, quedando la rama abierta si el árbol estuviera acabado y la sentencia sería satisfacible en un universo unitario. Como hipótesis de inducción: en el nivel $n$ existe una rama abierta $\mathcal{R}$ en la cual hay $k$ sentencias satisfechas por una $M^{\prime}$, diferente de $M$ a lo sumo respecto de la interpretación asignada a los parámetros que ocurren en dichas sentencias y no ocurren en $\Gamma^{\prime}$. Recorriendo las reglas que definen el árbol, se alcanza el resultado para $n+1$ nivel. 
En el otro sentido, si $\Gamma^{*}$ es el conjunto de todas las fórmulas de la rama abierta, en la cual ocurren $n \geq 1$ parámetros, se define una $L^{1}$-estructura de acuerdo con las siguientes cláusulas:

1. $D=\left\{b \in P A R\left(L^{1}\right): b\right.$ ocurre en alguna $\left.\beta \in \Gamma^{*}\right\}$.

2. $\Im(b)=b$, para todo $b \in D$.

3. $\Im(R)=\left\{<b_{1}, \ldots, b_{k}>\in D^{k}: R b_{1} \ldots b_{k} \in \Gamma^{*}\right\}$.

Fácilmente se verifica que esta $M=<D, \Im>$ satisface todas las sentencias de $\Gamma$, y, por definición, $|D|=n$. $\square$.

Teorema 5: Un conjunto de sentencias $\Gamma \subseteq L^{1}$ es no satisfacible si y sólo si el árbol de Beth de $\Gamma$ es cerrado.

La demostración se basa en el resultado de que en cada rama es definible una $L^{1}$-estructura que satisface todas las fórmulas de la rama. Si todas las ramas son cerradas, entonces en todas ocurren pares de contradicción y cada $L^{1}$-estructura definida a partir de las ramas habría de satisfacer estos pares, lo cual es absurdo. Por otra parte, si el conjunto es no satisfacible, entonces las ramas han de ser cerradas, pues de otro modo se entraría en contradicción con el resultado previo.

Podemos pasar ahora a aplicar estos resultados de primer orden a las clases de fórmulas de $L^{2}$ antes definidas. De las clases $\Pi_{1}^{1}$ y $\sum_{1}^{l}$ nos interesan las matrices, que son fórmulas esencialmente de primer orden. Previamente establecemos un resultado general:

Teorema 6: Cada sentencia de $L^{2} \sin$ constantes no lógicas es satisfacible en un dominio si y sólo si es válida en dicho dominio.

En efecto, si existe $M=\langle D, \Im\rangle$ tal que $M \models \varphi$, ello es independiente de los valores de la función interpretación, 
por lo que cualquiera que sea ésta, es decir, para cada $M^{\prime}=<D, \Im^{\prime}>, M^{\prime} \models \varphi$. $\square$.

Teorema 7: Para toda sentencia $\varphi \in \sum_{1}^{1}$ de la forma $\exists X_{1}, \ldots, X_{n} \psi$, sin relatores, $\models \varphi$ si y sólo si el árbol $\exists$-modificado de $\psi$ contiene una rama abierta en la que ocurre únicamente un parámetro.

Si $\models \exists X_{1}, \ldots, X_{n} \psi$, entonces la fórmula es válida en cada dominio $D$ tal que $|D| \geq 1$. Sea el caso en que $|D|=1$; entonces existe una $L^{1}$-estructura $M$ con $D$ como universo de discurso y tal que $M \models \psi$ y, por el teorema 4, en el árbol $\exists$-modificado hay una rama abierta con un número $|D|$ de parámetros, es decir 1. Recíprocamente, supongamos, en primer lugar, que en $\psi$ no ocurren parámetros: Si el árbol $\exists$-modificado de $\psi$ tiene una rama abierta con un único parámetro, entonces $\psi$ es 1-satisfacible, por el teorema 4 , y $m$-satisfacible, para todo $m \geq 1$, por el teorema 3 . Ahora bien, por evaluación de $\exists, \exists X_{1}, \ldots, X_{n} \psi$ es $m$-satisfacible para todo $m \geq 1$ y, por el teorema 6 , es válida en cada dominio de cardinal $m \geq 1$. En segundo lugar, si en $\psi$ ocurren parámetros, entonces se opera con el cierre existencial de $\psi$; sea ésta $\psi^{\prime}$, al obtener su árbol $\exists$-modificado puede suceder que no se den las circunstancias de la hipótesis, en cuyo caso $\psi^{\prime}$ no sería l-satisfacible y tampoco $\psi$, o bien $\psi^{\prime}$ es l-satisfacible y $\psi$ es satisfacible en un dominio unitario de tal manera que si $\Im$ es la función interpretación correspondiente, $\Im(a)=\Im(b)$ para cada par de parámetros $a, b$ que ocurran en $\psi$. Estamos así como en el caso anterior, es decir, la fórmula de $L^{2}$ es universalmente válida. $\square$.

Teorema 8: Para toda sentencia $\varphi \in \Pi_{1}^{1}$ de la forma $\forall X_{1}, \ldots, X_{n} \psi, \models \varphi$ si y sólo si el árbol de Beth de $\neg \psi$ es cerrado.

En efecto, si $\models \varphi$, entonces para toda $L^{1}$-estructura $M$, $M \models \psi$ (es, pues, universalmente válida), por lo que $\neg \psi$ es 
no satisfacible y, según el teorema 5 , ello equivale a afirmar que el árbol de Beth de $\neg \psi$ es cerrado. $\square$.

Respecto de las fórmulas de la clase $I Z$ también podemos establecer un resultado operando con árboles de primer orden, siempre que se introduzcan ligeras variantes de las nociones estudiadas. Consideremos una sentencia de esta clase en la que $\mathcal{Q}_{1}$ es vacío, es decir, tiene la forma $\mathcal{Q}_{2} \beta-\mathrm{O}$ bien es el resultado de haber aplicado las conocidas reglas de árboles de primer orden respecto de los cuantificadores de $\mathcal{Q}_{1}$-; entonces en $\beta$ no ocurre ningún cuantificador, los únicos signos lógicos que ocurren son las conectivas proposicionales. Con objeto de fijar el tratamiento de este tipo de sentencias, para cada fórmula $\beta$ y cada variable predicativa $n$-ádica, se define el conjunto de fórmulas $\Delta(\beta, X)$ y, tras modificar los árboles más arriba definidos, añadiendo reglas para la identidad, se pueden introducir unas reglas especiales para fórmulas resultantes de aplicar las reglas de árboles propias de primer orden. ${ }^{11}$

Teorema 9: Una sentencia de segundo orden de la forma $\mathcal{Q}_{1} \mathcal{Q}_{2} \beta$ es satisfacible si y sólo si el árbol de $\mathcal{Q}_{2} \beta^{*}$ es abierto (siendo esta última la sentencia obtenida desde $\mathcal{Q}_{1} \mathcal{Q}_{2} \beta$ sustituyendo en $\mathcal{Q}_{2} \beta$ cada variable individual libre — sometida a la cuantificación del prefijo $\mathcal{Q}_{1}$ - por el parámetro —o parámetros-, según las reglas correspondientes).

Para la demostración del teorema es necesario el siguiente

11 Para facilitar la lectura y teniendo en cuenta que estas nociones se usan en la demostración del lema enunciado más adelante, se incluyen en el apéndice. 
Lema: ${ }^{12}$ Dadas una sentencia $\mathcal{Q}^{\prime} \gamma$ siendo $\mathcal{Q} y \mathcal{Q}^{\prime}$ prefijos cuantificacionales predicativos, y una $L^{2}$-estructura $M$

1. Si $\mathcal{Q}$ es $\exists X, M \models \mathcal{Q}^{\prime} \gamma$ si y sólo si para todo $i \leq k$, $\delta_{i} \in \Delta(\gamma, X),|\Delta(\gamma, X)|=k$,

$$
M \models \mathcal{Q}^{\prime} \gamma\left(\delta_{1} / X\right) \vee \mathcal{Q}^{\prime} \gamma\left(\delta_{2} / X\right) \vee \ldots \vee \mathcal{Q}^{\prime} \gamma\left(\delta_{k} / X\right) ;
$$

2. Si $\mathcal{Q}$ es $\forall X, M \models \mathcal{Q} \mathcal{Q}^{\prime} \gamma$ si y sólo si para todo $i \leq k$, $\delta_{i} \in \Delta(\gamma, X),|\Delta(\gamma, X)|=k$,

$$
M \models \mathcal{Q}^{\prime} \gamma\left(\delta_{1} / X\right) \wedge \mathcal{Q} \gamma\left(\delta_{2} / X\right) \wedge \ldots \wedge \mathcal{Q}^{\prime} \gamma\left(\delta_{k} / X\right) .
$$

La demostración del Teorema 9 es análoga a la del Teorema 4, teniendo en cuenta este lema. $\square$.

5. Observaciones sobre estas aplicaciones

De acuerdo con la metodología expuesta, hemos extendido el procedimiento de árboles de primer orden, teniendo en cuenta la modificación incluida para el tratamiento de la clase de las $I Z$, de manera que podemos reseñar algunas consecuencias, para lo cual tendremos en cuenta los resultados anteriores y los siguientes.

Teorema 10: Para toda sentencia $\beta \in \Pi_{1}^{1}\left(\sum_{1}^{1}\right)$ existe otra sentencia $\beta^{*} \in \sum_{1}^{1}\left(\Pi_{1}^{1}\right)$, tales que $\models \beta$ si y sólo si $\beta^{*}$ es no satisfacible.

En efecto, sea $\beta$ de la forma $\forall X_{1}, \ldots, X_{m} \varphi, m \geq 1$. Obviamente, $\models \beta$ si y sólo si $\neg \beta$ es no satisfacible, pero

$$
\neg \forall X_{1}, \ldots, X_{m} \varphi \longleftrightarrow \exists X_{1}, \ldots, X_{m} \neg \varphi,
$$

12 Como en el caso de las nociones necesarias para la demostración, hemos incluido ésta en el apéndice. 
y $\exists X_{1}, \ldots, X_{m} \neg \varphi \in \sum_{1}^{l}$. De manera análoga, si $\beta$ es de la forma $\exists X_{1}, \ldots, X_{m} \varphi$, entonces $\beta^{*}$ sería $\forall X_{1}, \ldots, X_{m} \neg \varphi$.

Teorema 11: Para cada $\beta \in \Pi_{1}^{1}$ tal que $\forall Z_{1}, \ldots, Z_{k} \beta \in$ $\Pi_{1}^{1}$ y carezca variables libres, ${ }^{13}$ son equivalentes:

1. $\models \beta\left(R_{1}, \ldots, R_{k} / Z_{1}, \ldots, Z_{k}\right)$, siendo $R_{i}$ relator de la misma aridad que $Z_{i}$, para todo $i \leq k$;

2. $\models \forall Z_{1}, \ldots, Z_{k} \beta$;

3. si $\beta$ es $\forall X_{1}, \ldots, X_{t} \varphi, t \geq 1$, el árbol semántico de $\neg \varphi$ es cerrado.

La demostración es trivial a partir de la evaluación de $\forall$ y del Teorema 8. $\square$.

Teorema 12: Para cada $\beta \in \sum_{1}^{l}$ tal que $\exists Z_{1}, \ldots, Z_{k} \beta \in$ $\sum_{1}^{l} y$ carezca variables libres, son equivalentes:

1. $\beta\left(R_{1}, \ldots, R_{k} / Z_{1}, \ldots, Z_{k}\right)$ es satisfacible, siendo $R_{i}$ relator de la misma aridad que $Z_{i}$, para todo $i \leq k$;

2. $\exists Z_{1}, \ldots, Z_{k} \beta$ es satisfacible;

3. si $\beta$ es $\exists X_{1}, \ldots, X_{t} \varphi, t \geq 1$, el árbol semántico de $\varphi$ es abierto.

En efecto: (1) si y sólo si (2) por evaluación de $\exists$; (2) si y sólo si (3) si tenemos en cuenta el Teorema 4; (3) si y sólo si $\varphi$ es satisfacible (esencialmente de primer orden) si y sólo si lo es la sentencia

$$
\varphi\left(R_{1}, \ldots, R_{k}, S_{1}, \ldots, S_{t} / Z_{1}, \ldots, Z_{k}, X_{1}, \ldots, X_{t}\right),
$$

donde $S_{j}$ es relator de la misma aridad que $X_{j}$, para todo $j \leq t$, si bien ésta es satisfacible si y sólo si tores.

$13 Z_{1}, \ldots, Z_{k}$ son las variables libres de $\beta$, pudiendo ocurrir rela- 
$\exists X_{1}, \ldots, X_{t} \varphi\left(R_{1}, \ldots, R_{k} / Z_{1}, \ldots, Z_{k}\right)$, es satisfacible,

pero $\exists X_{1}, \ldots, X_{t} \varphi\left(R_{1}, \ldots, R_{k} / Z_{1}, \ldots, Z_{k}\right)$ es precisamente $\beta\left(R_{1}, \ldots, R_{k} / Z_{1}, \ldots, Z_{k}\right)$, luego (3) si y sólo si (1).

Respecto de las clases $\sum_{1}^{1}$ y $\Pi_{1}^{1}$ hemos obtenido resultados en parte esperados. Pero el estudio de la clase $I Z$ se puede considerar más novedoso. Van Benthen y Doets (1984) se habían ocupado de las primeras, aunque no de esta última; el lenguaje formal de primer orden que utilizan es un lenguaje con identidad. En cualquier caso, cabría considerar el método de los árboles alternativo a aquel que recurre a depuradas nociones de teoría abstracta de modelos (teoría de filtros: ultraproducto, ultrapotencia, colapso, etc.). Si bien nos hemos referido principalmente a sentencias en las cuales no ocurrían relatores, sino sólo variables predicativas ligadas, a partir de los últimos resultados es posible una ampliación de la aplicación del método. Asimismo, al operar sobre sentencias en forma prenexa con cuantificadores individuales precediendo a todos los cuantificadores predicativos, en el desarrollo de un árbol semántico, se obtienen sentencias de $I Z$, con las cuales se puede continuar si tenemos en cuenta lo estudiado más arriba.

Si la aplicabilidad del método de tablas semánticas en lógica de segundo orden no nos lleva a logros espectaculares, nos permite, al menos, alcanzar ciertos resultados desde otra perspectiva, lo que invita a continuar la reflexión sobre la naturaleza misma de la cuantificación, cómo se pueden plantear extensiones de lógica elemental, etc. En particular, si a partir del método de Beth es demostrable la completitud de los sistemas de cálculo de primer orden. ¿Qué se puede decir acerca de los sistemas de cálculo de segundo orden tras las modificaciones propuestas? La corrección 
de estos sistemas es un resultado conocido, también que la completitud es imposible como consecuencia del teorema de incompletitud de sistemas formales aritméticos de Gödel. ${ }^{14}$ No obstante, adoptando la nueva semántica propuesta por Henkin, ${ }^{15}$ es demostrable que los sistemas de cálculo de segundo orden son completos en sentido general.

Consideremos un sistema de cálculo tipo Hilbert (ampliado a segundo orden), que representamos por $\vdash$ (" $\vdash \alpha$ " indica que la fórmula $\alpha$ es demostrable en dicho cálculo). Adoptando la semántica estándar, la incompletitud de $\vdash$ podemos enunciarla de la siguiente manera: existe una sentencia $\gamma \in L^{2}$ tal que $\models \gamma$ pero no es el caso que $\vdash \gamma$; a partir de la fórmula aritmética construida por Gödel es definible una tal sentencia $\gamma$. No obstante, cabe esperar una cierta completitud, sin necesidad de cambiar la semántica estándar, siempre que sea relativa a una clase restringida de sentencias, que desde luego no contendrá fórmulas como la mencionada.

Sea la clase $\mathcal{F}_{\Delta} \subseteq L^{2}$, tal que verifica

1. Si $\varphi \in L^{2}$ y $\varphi \in \sum_{1}^{1} \cup \Pi_{1}^{1} \cup I Z$, entonces $\varphi \in \mathcal{F}_{\Delta}$.

2. Si $\psi$ es la forma prenexa de $\varphi$ y $\psi \in \mathcal{F}_{\Delta}$, entonces $\varphi \in \mathcal{F}_{\Delta}$.

Respecto de esta clase, y teniendo en cuenta los resultados anteriores, se podrá establecer que para cada $\varphi \in \mathcal{F}_{\Delta}$, si $\models \varphi$, entonces $\vdash \varphi$, y, dada corrección de $\vdash$,

14 Manzano (1996) ha presentado una prueba directa de incompletitud, al margen del teorema de Gödel; dicha prueba está basada en ciertas técnicas de teoría de conjuntos y en la capacidad expresiva que caracteriza la lógica de segundo orden.

15 En Henkin (1950). Esquemáticamente, en sentido general, en las estructuras interpretativas se tienen en cuenta el universo de discurso (no vacío) y también universos relacionales (para cada aridad), los cuales poseen ciertas características algebraicas, como se describe en Manzano (1996), lo cual equivale a que todas las relaciones son definibles (con el lenguaje formal de que se trate). 
para cada $\varphi \in \mathcal{F}_{\Delta}, \models \varphi$ si y sólo si $\vdash \varphi$.

\section{Apéndice}

Consideramos ahora el lenguaje formal $L^{2=}$, obtenido a partir de $L^{2}$ con el relator diádico $=$ y considerando que si $a$ y $b$ son variables individuales o parámetros, $a=b \in$ $L^{2=}$. Para mayor facilidad, se escribirá $a \neq b$ en lugar de $\neg(a=b)$. Por lo que respecta a la semántica de $L^{2=}$, si $M=<D, \Im>$, para cualesquiera parámetros $a$ y $b$, $M \models a=b$ si y sólo si $\Im(a)=\Im(b){ }^{16}$

Definimos, para $\beta$ y cada variable predicativa $n$-ádica $X$, el conjunto de fórmulas $\Delta(\beta, X)$ por inducción sobre el grado de complejidad de $\beta$ (para simplificar, consideraremos que $\neg \mathrm{y} \vee$ son las únicas conectivas que ocurren en $\beta) . \Delta(\beta, X)$ es el más pequeño conjunto que verifica:

1. Si en $\beta$ no ocurre $X$, entonces $\Delta(\beta, X)=\emptyset$.

2. Si $\beta$ es $X b_{1} \ldots b_{n}$, entonces $\Delta(\beta, X)=\{\delta, \neg \delta\}$, en donde $\delta$ es $x_{1}=b_{1} \wedge \ldots \wedge x_{n}=b_{n}$. Por otra parte, se dice que $\delta$ es positiva, y $\neg \delta$ negativa.

3. Si $\beta$ es $\neg \gamma$, entonces $\Delta(\beta, X)=\Delta(\gamma, X)$.

4. Si $\beta$ es $\gamma \vee \eta$, entonces, $\Delta(\beta, X)$ es el mínimo conjunto tal que:

i. Si $\theta \in \Delta(\gamma, X)$ o $\theta \in \Delta(\eta, X)$ y $\theta$ es positiva, entonces $\theta \in \Delta(\beta, X)$.

ii. Para toda $i, j \geq 1$, si $\theta_{i} \in \Delta(\gamma, X)$ o $\theta_{j} \in \Delta(\eta, X)$ $\mathrm{y}$ ambas son positivas, entonces $\theta_{i} \vee \theta_{j} \in \Delta(\beta, X)$.

iii. Para $n>1$, toda $i \leq n$, si $\vartheta_{i} \in \Delta(\gamma, X)$ o $\vartheta_{i} \in$ $\Delta(\eta, X)$ y $\vartheta_{i}$ es negativa, entonces $\vartheta_{1} \wedge \ldots \wedge \vartheta_{n} \in$ $\Delta(\beta, X)$.

16 Fácilmente se comprueba que $\models a=b \leftrightarrow \forall X(X a \leftrightarrow X b)$. 
5. Si $\beta$ es $Q \gamma$, donde $Q$ es un prefijo cuantificacional cuyos sufijos son $Z_{1}, \ldots, Z_{m}$, las $m \geq 1$ variables predicativas distintas de $X$ que ocurren en $\gamma$, entonces

$$
\Delta(Q \gamma, X)=\Delta\left(\beta\left(R_{1}, \ldots, R_{m} / Z_{1}, \ldots, Z_{m}\right), X\right),
$$

siendo $R_{i}$ un relator de la misma aridad que $Z_{i}$, para toda $i \leq m$.

Los árboles más arriba definidos se pueden modificar añadiendo:

1. Si en una rama ocurre una sentencia de la forma $b \neq$ $b$, en donde $b$ es un parámetro cualquiera, entonces se produce el mismo efecto que si se tratara de un par de contradicción; es decir, la rama es cerrada.

2. Si en una rama ocurren fórmulas de la forma $\beta\left(a_{j} / x\right)$ y $a_{i}=a_{j}$, siendo éstos unos parámetros tales que $i \leq j$, entonces se tachan todas las fórmulas $\beta\left(a_{j} / x\right)$ $\mathrm{y}$ se sustituyen por $\beta\left(a_{i} / x\right)$ (también se pueden tachar las mencionadas fórmulas de identidad).

Dada una fórmula de la clase $I Z$, que tendrá, por tanto, la forma $\mathcal{Q}_{1} \mathcal{Q}_{2} \beta$, en donde $\beta$ es esencialmente de primer orden, y teniendo en cuenta la modificación de las reglas de los árboles respecto de fórmulas de identidad, se definen las siguientes nuevas reglas específicas para el prefijo $\mathcal{Q}_{2}$ (para la construcción de árboles tanto de Beth, como $\exists$ modificados, aunque ya específicos de segundo orden). Sea un punto del árbol en que la fórmula a marcar tiene la forma $\mathcal{Q}_{2} \beta$ y $\beta$ no contiene variables individuales; para mayor facilidad consideremos que $\left|\mathcal{Q}_{2}\right|=1 \mathrm{y}$, por tanto, únicamente ocurre un cuantificador predicativo, entonces

1. Si $\mathcal{Q}_{2} \beta$ es $\exists X \beta, X$ variable predicativa de aridad $n \geq 1$, entonces se abren subramas cada una con 


$$
\beta\left(\rho_{i}\left(b_{1}, \ldots, b_{n} / x_{1}, \ldots, x_{n}\right) / X b_{1}, \ldots, b_{n}\right),
$$

para toda $\rho_{i} \in \Delta(\beta, X)$, marcándose la fórmula inicial con dichas $\rho_{i}$ como subíndices.

2. Si $\mathcal{Q}_{2} \beta$ es $\forall X \beta, X$ variable predicativa de aridad $n \geq 1$, entonces se añaden a la rama las fórmulas

$$
\beta\left(\rho_{i}\left(b_{1}, \ldots, b_{n} / x_{1}, \ldots, x_{n}\right) / X b_{1}, \ldots, b_{n}\right),
$$

para toda $\rho_{i} \in \Delta(\beta, X)$, marcándose la fórmula inicial con dichas $\rho_{i}$ como subíndices.

Demostración del Lema: [Inducción sobre el grado de complejidad de $\mathcal{Q}^{\prime} \gamma$.] Comencemos considerando el caso en que $\mathcal{Q}^{\prime}=\emptyset$ :

1. $\gamma$ es $X b_{1} \ldots b_{n}$, para $n \geq 1$. En tal caso, para cada $L^{2}$-estructura $M$

$\bullet M \models\left(b_{1}=b_{1} \wedge \ldots \wedge b_{n}=b_{n}\right) \vee\left(b_{1} \neq b_{1} \vee \ldots \vee b_{n} \neq\right.$ $b_{n}$ ), asimismo $M \models \exists X \gamma$ (ambas son válidas en el correspondiente dominio);

- $M$ no satisface $\left(b_{1}=b_{1} \wedge \ldots \wedge b_{n}=b_{n}\right) \wedge\left(b_{1} \neq\right.$ $\left.b_{1} \vee \ldots \vee b_{n} \neq b_{n}\right)$, pero $M$ no satisface $\forall X \gamma$ tampoco.

2. $\gamma$ es $\neg \eta$; por hipótesis, $M \models \exists X \eta \Longleftrightarrow^{17} M \models$ $\eta\left(\delta_{1} / X\right) \vee \ldots \vee \eta\left(\delta_{k} / X\right)$, para $\delta_{i} \in \Delta(\eta X), i \leq k$ y $k \geq 1$; asimismo, $M \models \forall X \eta \Longleftrightarrow M \models \eta\left(\delta_{1} X\right) \wedge$ $\ldots \wedge \eta\left(\delta_{k} X\right)$, para $\delta_{i} \in \Delta(\eta X), i \leq k$ y $k \geq 1$; además $\Delta(\gamma, X)=\Delta(\eta, X)$. Entonces, $M \models \exists X \neg \eta \Longleftrightarrow$ $M \models \neg \forall X \eta \Longleftrightarrow \operatorname{no}-(M \models \forall X \eta) \Longleftrightarrow \operatorname{no}(M \models$ $\left.\eta\left(\delta_{1} / X\right) \wedge \ldots \wedge \eta\left(\delta_{k} / X\right)\right) \Longleftrightarrow M \models \neg \eta\left(\delta_{1} / X\right) \vee \ldots \vee$

17 Abreviatura que indica la equivalencia entre las proposiciones anotadas a sus extremos. Es decir, $\mathrm{A} \Longleftrightarrow \mathrm{B}$, representa "si $\mathrm{A}$, entonces $\mathrm{B}$, y si B, entonces $\mathrm{A}^{\prime \prime}$. 
$\neg \eta\left(\delta_{k} / X\right)$. Recíprocamente, $M \models \forall X \neg \eta \Longleftrightarrow M \models$ $\neg \exists X \eta \Longleftrightarrow$ no- $(M \models \exists X \eta) \Longleftrightarrow$ no- $\left(M \models \eta\left(\delta_{1} / X\right) \vee\right.$ $\left.\ldots \vee \eta\left(\delta_{k} / X\right)\right) \Longleftrightarrow M \models \neg \eta\left(\delta_{1} / X\right) \wedge \ldots \wedge \neg \eta\left(\delta_{k} / X\right)$.

3. $\gamma$ es $\eta \vee \theta$. Establecemos, según la definición dada más arriba, $\Delta(\gamma, X)=\Delta^{*}$, es el más pequeño conjunto tal que:

i) si $\delta \in \Delta(\eta, X)$ ó $\delta \in \Delta(\theta, X)$ y $\delta$ es positiva, entonces $\delta \in \Delta^{*}$;

ii) para todo $i, j$, si $\delta_{i} \in \Delta(\eta, X), \delta_{j} \in \Delta(\theta, X)$ y ambas son positivas, $\delta_{i} \vee \delta_{j} \in \Delta^{*}$;

iii) para todo $i, j$, si $\delta_{i} \in \Delta(\eta, X), \delta_{j} \in \Delta(\theta, X)$ y ambas son negativas, $\delta_{i} \wedge \delta_{j} \in \Delta^{*}$.

Se nos presentan dos casos:

(a) $M \models \forall X(\eta \vee \theta)$, entonces para toda asignación $s$ de valores a las variables se verifica que $M, s \models \eta \vee \theta ;^{18}$ entonces, para cada $\delta_{k} \in \Delta(\eta / X), \delta_{m} \in \Delta(\theta / X)$, $M \models \eta\left(\delta_{k} / X\right)$ ó $M \models \theta\left(\delta_{m} / X\right)$. Si $\delta \in \Delta^{*}$ por i), $M \models \eta(\delta / X)$ ó $M \models \theta(\delta / X)$; si $\delta \in \Delta^{*}$ es $\delta_{k} \vee \delta_{m}$, definida por ii), $M \models \eta\left(\delta_{k} \vee \delta_{m} / X\right)$ ó $M \models$ $\theta\left(\delta_{k} \vee \delta_{m} / X\right)$; si $\delta \in \Delta^{*}$ es $\delta_{k} \wedge \delta_{m}$ definida según iii), $M \models \eta\left(\delta_{k} \wedge \delta_{m} / X\right)$ ó $M \models \theta\left(\delta_{k} \wedge \delta_{m} / X\right)$. Es decir, para cada $\delta \in \Delta^{*}, M \models \eta(\delta / X) \vee \theta(\delta / X)$, luego $M \models(\eta \vee \theta)(\delta / X)$; si $\left|\Delta^{*}\right|=r, M \models(\eta \vee \theta)\left(\delta_{1} / X\right) \wedge$ $\ldots \wedge(\eta \vee \theta)\left(\delta_{r} / X\right)$. Recíprocamente, si $\left|\Delta^{*}\right|=r$, para $\delta_{i} \in \Delta^{*}, i \leq r, M \models(\eta \vee \theta)\left(\delta_{1} / X\right) \wedge \ldots \wedge(\eta \vee$ $\theta)\left(\delta_{r} / X\right)$, entonces $M \models\left(\eta\left(\delta_{1} / X\right) \vee \theta\left(\delta_{1} / X\right)\right) \wedge \ldots \wedge$ $\left(\eta\left(\delta_{r} / X\right) \vee \theta\left(\delta_{r} / X\right)\right)$, de donde, para toda asignación $s$, $M, s \models \eta \vee \theta$, y, por evaluación de $\forall, M \models \forall X(\eta \vee \theta)$.

18 En el caso básico, $M, s \models X b_{1}, \ldots, b_{n}$ para toda asignación $s$ si y sólo si $M \models\left(b_{1}=b_{1} \wedge \ldots\right) \wedge\left(b_{1} \neq b_{1} \wedge \ldots\right)$ (ninguna es satisfecha por esta estructura). Por inducción, se establece que $M, s \models \gamma$, para toda asignación $s$, si y sólo si $M \models \gamma\left(\delta_{1} / X\right) \wedge \ldots \wedge \gamma\left(\delta_{r} X\right)$ para toda $\delta_{i} \in \Delta(\gamma, X),|\Delta(\gamma, X)|=r$. 
(b) $M \models \exists X(\eta \vee \theta)$, entonces para alguna asignación $s$, $M, s \models \eta \vee \theta$; de manera análoga al caso precedente, hay alguna $\delta \in \Delta^{*}$ tal que $M \models(\eta \vee \theta)(\delta / X)$, es decir, $M \models \eta(\delta / X) \vee \theta(\delta / X)$. Teniendo en cuenta la construcción de $\Delta^{*}$, se alcanza que $M \models(\eta \vee$ $\theta)\left(\delta_{1} / X\right) \vee \ldots \vee(\eta \vee \theta)\left(\delta_{r} / X\right)$. Recíprocamente, para $\delta_{i} \in \Delta^{*}, i \leq r$, si $M \models(\eta \vee \theta)\left(\delta_{1} / X\right) \vee \ldots \vee(\eta \vee$ $\theta)\left(\delta_{r} / X\right)$, entonces $M \models\left(\eta\left(\delta_{1} / X\right) \vee \theta\left(\delta_{1} / X\right)\right) \vee \ldots \vee$ $\left(\eta\left(\delta_{r} / X\right) \vee \theta\left(\delta_{r} / X\right)\right)$, de donde, para alguna asignación $s$, se verifica $M, s \models \eta \vee \theta$, y, por evaluación de $\exists, M \models \exists X(\eta \vee \theta)$.

Consideremos ahora que $\left|\mathcal{Q}^{\prime}\right|=m \geq 1$, siendo $Z_{1}, \ldots, Z_{m}$ las variables (predicativas) sufijo de los $m$ cuantificadores que integran $\mathcal{Q}^{\prime}$. Habremos de tener en cuenta la cláusula 5 que define $\Delta\left(\mathcal{Q}^{\prime} \gamma, X\right)=\Delta\left(\gamma\left(R_{1}, \ldots, R_{m} /\right.\right.$ $\left.\left.Z_{1}, \ldots, Z_{m}\right), X\right)$; llamamos $\gamma^{\prime}$ a la fórmula $\gamma\left(R_{1}, \ldots, R_{m} /\right.$ $\left.Z_{1}, \ldots, Z_{m}\right)$. Entonces:

1. $M \models \exists X \mathcal{Q}^{\prime} \gamma$; entonces para alguna asignación $s$, $M, s \models \mathcal{Q}^{\prime} \gamma$; para cada $\delta_{i} \in \Delta\left(\gamma^{\prime}, X\right), i \leq r$, asignación $s^{\prime}$ (que difiere de $s$ a lo sumo con respecto de los valores de $\left.R_{1}, \ldots, R_{m}\right), M, s^{\prime} \models \gamma^{\prime}\left(\delta_{1} / X\right) \vee$ $\ldots \vee \gamma^{\prime}\left(\delta_{r} / X\right) .{ }^{19}$ Fácilmente (iterando el caso de disyunción visto anteriormente) se alcanza que $M, s \models$ $\mathcal{Q}^{\prime} \gamma\left(\delta_{1} / X\right) \vee \ldots \vee \mathcal{Q}^{\prime} \gamma\left(\delta_{r} / X\right)$ (asimismo, recíprocamente).

2. $M \models \forall X \mathcal{Q}^{\prime} \gamma$; entonces para toda asignación $s$, $M, s \models \mathcal{Q}^{\prime} \gamma$; para cada $\delta_{i} \in \Delta\left(\gamma^{\prime}, X\right), i \leq r$, asignación $s^{\prime}$ (que difiere de $s$ a lo sumo con respecto de los valores de $\left.R_{1}, \ldots, R_{m}\right), M, s^{\prime} \models \gamma^{\prime}\left(\delta_{1} / X\right) \wedge$

19 En este apartado, como en el siguiente, cabría distinguir varios casos: $\mathcal{Q}^{\prime}=\forall Z_{1} \forall Z_{2}$, o $\mathcal{Q}^{\prime}=\forall Z_{1} \exists Z_{2}$, o $\mathcal{Q}^{\prime}=\exists Z_{1} \forall Z_{2}$, о $\mathcal{Q}^{\prime}=\exists Z_{1} \exists Z_{2}$ (con lo que $m=2$ ); supuesto para $\mathcal{Q}^{*}$, estudiar el de $\mathcal{Q}^{\prime}=\mathcal{Q}^{*} \forall Z_{m}$ o $\mathcal{Q}^{\prime}=\mathcal{Q}^{*} \exists Z_{m}$, teniendo siempre en cuenta el modo de evaluación de los cuantificadores (cuyo detalle omitimos para abreviar). 
$\ldots \wedge \gamma^{\prime}\left(\delta_{r} / X\right)$. Fácilmente (teniendo en cuenta el caso de disyunción también visto antes) se alcanza que $M, s \models \mathcal{Q}^{\prime} \gamma\left(\delta_{1} / X\right) \wedge \ldots \wedge \mathcal{Q}^{\prime} \gamma\left(\delta_{r} / X\right)$ (asimismo, recíprocamente).

\section{BIBLIOGRAFÍA}

van Benthem, J. y K. Doets, 1984, "Higher-Order Logic", Handbook of Philosophical Logic, Gabbay y Genthner (comps.), Kluwer Adademic Publishers, Dordrecht, vol. I, pp. 275-330.

Beth, E.W., 1955, "Semantic Entailment and Formal Derivability", en J. Hintikka (1969), The Philosophy of Mathematics, Oxford University Press Londres. [Traducido en Cuadernos Teorema, no. 18, "Entrañamiento semántico y derivabilidad formal", Valencia, 1978.]

Boolos, G., 1984, "Trees and Finite Satisfactibility", Notre Dame Journal of Formal Logic, vol. 25, pp. 110-115. Díaz, E., 1993, “Árboles semánticos y modelos mínimos”, Madrid, Actas del I Congreso de la Sociedad de Lógica, Metodología y Filosofía de la Ciencia en España, pp. 40-43.

— 1987, "Conjuntos enumerables representativos de conjuntos no enumerables", Colloquium 1985-1986, Badajoz, vol. 15, pp. 51-66.

Henkin, L., 1950, "Completeness in The Theory of Types", en Hintikka (1969), pp. 53-63.

— 1949, "The Completeness of The First-Order Functional Calculus", en Hintikka (1969), pp. 42-52.

Hilbert, D. y W. Ackerman, 1962, Elementos de lógica teórica. [Trad. V. Sánchez de Zabala, $6^{a}$ edición), Tecnos, Madrid.

Manzano, M., 1996, Extensions of First Order Logic, Cambridge University Press, Cambridge. 
Nepomuceno, A., 1995, "Lógica formal elemental", en A. Nepomuceno (comp.), Lógica formal. Orígenes, métodos y aplicaciones, Kronos, 1995, pp. 37-66.

Zykov, A.A., 1956, "The Spectrum Problem in The Extended Predicate Calculus", Translations of American Mathematical Society, vol. 3, pp. 1-14.

Recibido: 8 de diciembre de 1999 


\section{SUMMARY}

Beth's method of semantic tableaux has been utilized in first order logic to obtain some results. From a metalogical point of view, a known result can be proved: completeness of first order logic. Can we extend this method to second order logic? What about completeness? Given a second order formal language, for every formula a prenex form can be defined so that some of them have a first order formula as its matrix, then the mentioned method could be applied to study several classes of formulae, particularly $\sum_{1}^{l}, \Pi_{1}^{1}$ and Zykov's inverses. In order to do that some modifications of such method must be introduced, so that we take known rules for first order semantic trees, by applying them, modifying the treatment of " $\exists$ " and defining specific rules for some second order formulae, then we can achieve some results relative to such classes similar to that obtained by means of abstract model theory. On the other hand, a restricted completeness (to certain class of sentences) could be settled. 